\title{
Ausência de raça e gênero no enfrentamento da pandemia no Brasil
}

\author{
Absence of race and gender in the fight against the pandemic in Brazil \\ Falta de raza y género en el enfrentamiento a la pandemia en Brasil
}

Ivonete da Silva Lopes ${ }^{1, a}$

ivonetelopes@ufv.br | https://orcid.org/oooo-0001-5162-4719

\footnotetext{
${ }^{1}$ Universidade Federal de Viçosa, Departamento de Economia Rural, Programa de Pós-Graduação em Extensão Rural. Viçosa, MG, Brasil.

a Doutorado em Comunicação pela Universidade Federal Fluminense.
}

\section{RESUMO}

Esta nota discute a ausência dos quesitos cor/raça e gênero nos boletins epidemiológicos da Covid-19 e como essa omissão está articulada ao modo como se operacionaliza o racismo no Brasil. Coloca-se em evidência a apropriação da identidade racial e de gênero por alguns estados brasileiros que ganharam visibilidade midiática, como sendo solidários, ao iniciarem a campanha de vacinação com mulheres negras, grupo social mais vulnerável. É o jogo da dissimulação, em que se evidencia a raça/gênero na aparência, embora essas variáveis não sejam consideradas no enfrentamento da pandemia.

Palavras-chave: Covid-19; Mulheres negras; Racismo; Gênero; Desigualdades sociais.

\section{ABSTRACT}

This note discusses the non-inclusion of information about color/race and gender in Covid-19 epidemiological reports and how this omission is related to the way that the racism is operated in Brazil. It highlights the racial and gender identity appropriation by some Brazilian states, which have gained media visibility as sympathetic governments to Black women, by starting the vaccination campaign with them, an extremely vulnerable social group. It is like a confidence trick, in which race and gender are in the spotlight, although these features are not considered in the fight against the pandemic.

Keywords: Covid-19; Black women; Racism; Gender, Social inequities. 
Reciis - Revista Eletrônica de Comunicação, Informação \& Inovação em Saúde, Rio de Janeiro, v. 15, n. 2, p. 294-300, abr./jun. 2021 [www.reciis.icict.fiocruz.br] e-ISSN 1981-6278

\section{RESUMEN}

Esta nota discute la ausencia de las informaciones sobre cor/raza y género en los boletines epidemiológicos de la Covid-19 y como esa omisión se encuentra articulada al modo en que se opera el racismo en Brasil. Destaca la apropiación de la identidad racial y de género por parte de algunos estados brasileños que han ganado visibilidad mediática, vistos como solidarios, puesto que han iniciado la campaña de vacunación con algunas mujeres negras, el grupo social más vulnerable. Es el juego del disimulo, por lo cual es evidenciada la raza/género en la apariencia, sin embargo esas variables no sean tenidas en cuenta en el enfrentamiento a la pandemia.

Palabras clave: Covid-19; Mujeres negras; Racismo; Género; Desigualdades sociales.

Contribuição dos autores: texto integralmente redigido pela autora.

Declaração de conflito de interesses: não há

Fontes de financiamento: não houve.

Considerações éticas: não há.

Agradecimentos/Contribuições adicionais: agradeço à bolsista de Iniciação Científica CNPq, Jéssica Suzana Cardoso Magalhães, pela coleta e conferência dos dados dos websites governamentais, e à doutoranda no Programa de PósGraduação em Extensão Rural/UFV, Daniela Leal, pela revisão do texto.

Histórico do artigo: submetido: 2 jun. 2021 | aceito: 2 jun. 2021 | publicado: 30 jun. 2021.

Apresentação anterior: não houve.

Licença CC BY-NC atribuição não comercial. Com essa licença é permitido acessar, baixar (download), copiar, imprimir, compartilhar, reutilizar e distribuir os artigos, desde que para uso não comercial e com a citação da fonte, conferindo os devidos créditos de autoria e menção à Reciis. Nesses casos, nenhuma permissão é necessária por parte dos autores ou dos editores. 


\section{INTRODUÇÃO}

Uma mulher negra, a enfermeira Mônica Calazans, 54 anos, foi a primeira imunizada contra a Covid-19 em São Paulo e ganhou visibilidade por marcar a abertura da campanha nacional de vacinação. Outros estados adotaram o mesmo padrão e escolheram uma mulher negra para dar início ao processo de imunização local. O que há de errado? Nada, se considerarmos que os negros (pretos e pardos) correspondem a 56,4\% da população brasileira, e as mulheres negras constituem o grupo mais vulnerável. Somam $28 \%$ dessa população, mas estão sobrerrepresentadas na pobreza correspondendo a 32,8\%, e são 9,1\% dos brasileiros que vivem na extrema pobreza (SARAIVA, 2020). ${ }^{\mathrm{i}}$

Discuto nesta nota de conjuntura a contradição entre a aparência, a estratégia de comunicação adotada no lançamento da vacinação, e a ausência da informação sobre gênero e raça/cor nos boletins epidemiológicos divulgados diariamente pelos estados e pelo governo federal. Cabe destacar que as comunidades negras são mais afetadas pela pandemia por sofrerem com desigualdades estruturais e conviverem com riscos preexistentes, como: moradias inadequadas, alimentação pobre em nutrientes, dificuldade de acesso às medidas sanitárias, e doenças crônicas (ETOWA; HYMAN, 2021; AHMED, 2020; STARR; O'CONNOR; MEGHANI, 2021; GOMES, 2020; CALDWELL; ARAÚJO, 2020). Essas vulnerabilidades limitam as escolhas individuais e deveriam ser foco das políticas de enfrentamento da pandemia.

A coleta das informações sobre os casos e óbitos em decorrência da Covid-19 por etnia e raça e gênero são medidas para identificar a relação entre racismo, sexismo e o reflexo deles no acesso à saúde (ONU, 2020). No Brasil, a coleta interseccional de dados na área da saúde tem sido destacada como fundamental para que se possa pensar e desenvolver políticas mais efetivas no setor, como apontam as pesquisadoras Lopes e Werneck (2009):

O uso do quesito cor na intersecção com outras variáveis tais como sexo, idade, ocupação, escolaridade, local de moradia, entre outras, é essencial para identificar necessidades e demandas, definir prioridades, alocar recursos e promover mudanças nos processos de trabalho (p. 20).

O mais relevante a ser observado é a existência de dois dispositivos legais que determinam a coleta desagregada por cor, etnia e gênero. Um deles é a Política Nacional de Saúde Integral da População Negra (BRASIL, 2009), de 2009, documento no qual o Estado reconhece o racismo institucional e as desigualdades étnico-raciais dele resultantes e prevê várias medidas para promover a saúde dessa população. O segundo é o Estatuto da Igualdade Racial (Lei 12.888/2010), que trata sobre o tema no seu artigo $8^{\circ}$, ao prever "a melhoria da qualidade dos sistemas de informação do SUS no que tange à coleta, ao processamento e à análise dos dados desagregados por cor, etnia e gênero." (BRASIL, 2019, p. 13).

\section{COR/RAÇA E GÊNERO NOS BOLETINS EPIDEMIOLÓGICOS}

No início da pandemia, os dados de casos de infecção pelo vírus e de mortes pela doença eram divulgados sem especificações de cor/raça. A ausência dessas informações resultou em uma carta assinada por 150 entidades representativas do Movimento Negro e das periferias do país, integrantes da Coalizão Negra por Direitos, que foi enviada ao então ministro da Saúde, Luiz Henrique Mandetta. O documento solicitava que as informações sobre cor/raça, etnia, gênero e território fossem divulgadas (GOMES, 2020). Entretanto, as publicações do governo federal com a adoção das variáveis solicitadas não tiveram continuidade, ficando sob a responsabilidade dos estados a decisão de coletarem, ou não, e publicizarem esses dados. Uma incongruência do governo federal se considerada a existência de dispositivos legais que indicam a necessidade da coleta e do processamento dessas informações (BRASIL, 2009, 2019).

i O Brasil segue o padrão do Banco Mundial, ou seja, são considerados pobres aqueles que vivem com menos de 5,50 dólares por dia, e extremamente pobre os que sobrevivem com menos de 1,90 dólar. 
A omissão do governo federal na orientação aos entes da federação tem resultado na pouca coleta e no insuficiente processamento da estatística sobre raça/cor e gênero, conforme aponta o levantamento, realizado em abril de 2021, nos boletins epidemiológicos publicados em todos os 26 estados da federação e no Distrito Federal.

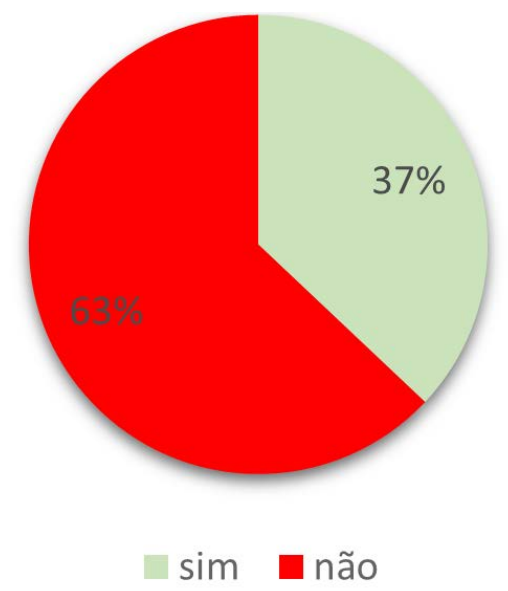

Figura 1 - Informação sobre a raça no boletim epidemiológico dos estados brasileiros Fonte: elaboração da autora.

A maioria dos estados (17) não divulga informação sobre pertencimento étnico-racial nos boletins epidemiológicos diários. Se desagregados esses dados por região, temos a Sul como a mais omissa neste quesito, uma vez que nenhum de seus três estados traz a estatística. Do lado oposto aparece a Região Norte, onde quatro dos seis estados apresentam raça na comunicação dos dados. No Nordeste, apenas três dos nove estados o fazem; no Sudeste, dois estados; e, no Centro-Oeste, apenas um ${ }^{\text {ii }}$. Comparando os dados desta pesquisa com a realizada por Santos et al., em junho de 2020, percebe-se que duplicou o número de estados que passaram a incorporar raça na coleta e divulgação dos dados sobre Covid-19. De cinco, em 2020, passaram a ser 10 estados no ano seguinte.

Quando se observa a coleta de dados desagregados por gênero e pertencimento étnico-racial, temos $15 \%$ dos estados que divulgam essa informação, ou seja, apenas quatro das 27 unidades da federação. São eles: Amazonas, Acre, Roraima e Espírito Santo. Assim como na produção de estatística sobre cor/raça, a Região Norte também aparece na coleta de dados por sexo como a mais representativa, já que três de seis estados da região os especificam.

ii Divulgam raça no boletim diário: Acre, Amapá, Amazonas, Bahia, Espírito Santo, Maranhão, Mato Grosso, Minas Gerais, Rio Grande do Norte e Roraima. 


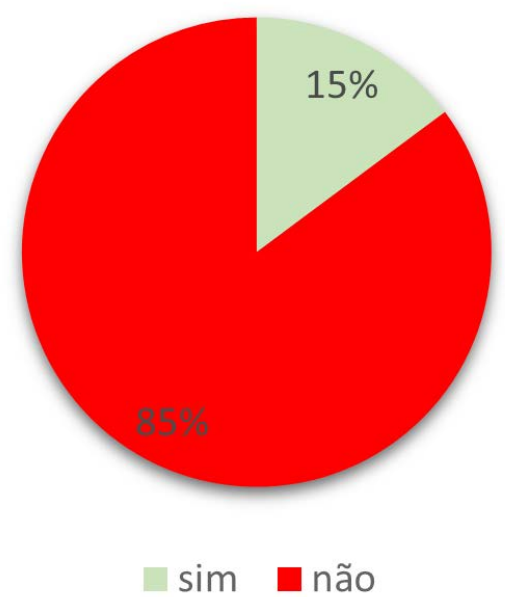

Figura 2 - Estados brasileiros e coleta de dados Covid-19 por cor/raça e sexo Fonte: elaboração da autora.

Apesar da resistência verificada no Brasil à produção e divulgação de dados do perfil sobre gênero e pertencimento racial relacionado ao contágio e aos óbitos pela Covid-19, é importante destacar como negros e mulheres negras figuraram como as primeiras pessoas imunizadas na maioria dos estados. Tomamos o Sudeste como emblemático para se discutir como gênero e raça são apropriados para agregar valor aos discursos ou às estratégias de marketing social/político. Nessa região, apenas Minas Gerais e Espírito Santo divulgam informação diária sobre pertencimento racial e Covid-19, e o último estado é o único do Sudeste que traz estatísticas sobre gênero e raça/cor no boletim epidemiológico diário, inclusive identificando as etnias indígenas ${ }^{\text {iii }}$. Contudo, todos os estados dessa região optaram por uma mulher negra para dar início ao processo de imunização, conforme se observa nas imagens (Figura 3) capturadas dos websites governamentais.
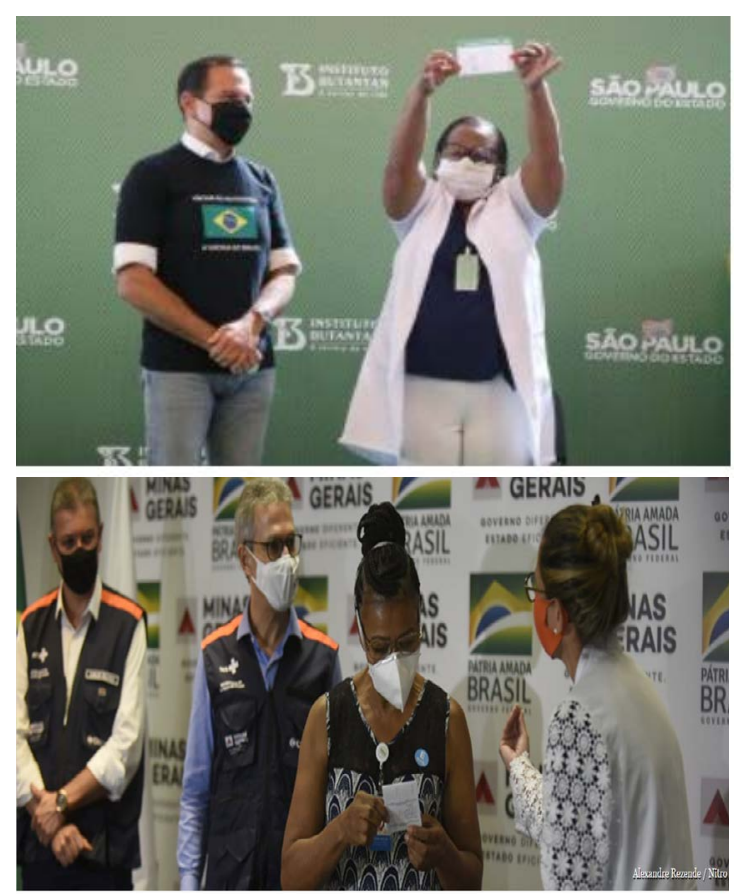
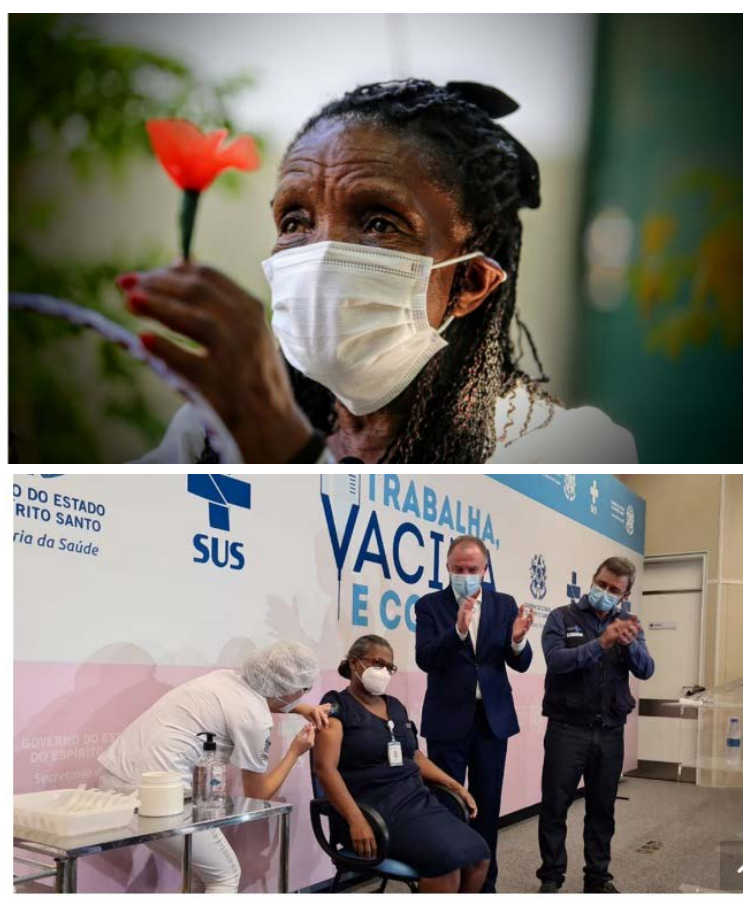

Figura 3 - Vacinação no Brasil e na Região Sudeste: Mônica Calazans (São Paulo), Terezinha da Conceição (Rio de

iii Minas Gerais e São Paulo trazem dados sobre gênero e raça apenas nos boletins acumulados, contudo não ficam facilmente disponível nos sites. 
Janeiro), Maria Bom Sucesso Pereira (Minas Gerais) e Iolanda Brito (Espírito Santo)

Fonte: PRIMEIRA..., 2021; COVID..., 2021; MINAS..., 2021; GOVERNO..., 2021.

Em três estados (São Paulo, Minas Gerais e Espírito Santos), a estratégia adotada coloca os governadores, homens brancos, ao lado das mulheres negras. Como ler essas imagens? Será que podemos associá-las à imagem (mito) do branco-salvador tão difundida nas produções audiovisuais? Continuando a análise, entre os textos da comunicação governamental que dão suporte às imagens, somente o publicado pelo governo paulista faz menção ao gênero e à raça. Ele destaca ser a vacinada: "Mulher, negra e com perfil de alto risco [obesa, hipertensa e diabética] para complicações provocadas pelo coronavírus, não deixou de atuar nos hospitais da capital paulista para ajudar a salvar vidas" (PRIMEIRA..., 2020).

A publicação conta um pouco da trajetória da enfermeira, que atuou por 26 anos como auxiliar de enfermagem, entrou tardiamente na universidade e aos 47 anos de idade conseguiu o diploma de ensino superior. Apesar da formação em enfermagem, trabalha de forma precarizada com contrato temporário em hospital na capital paulistana. Em resumo, a vida de Mônica retrata os riscos preexistentes com os quais convive a população negra e as dificuldades de ascensão social, como se observa no depoimento da enfermeira: "Não é apenas uma vacina. É o recomeço de uma vida que pode ser justa, sem preconceitos e com garantia de que todos nós teremos as mesmas condições de viver dignamente, com saúde e bem-estar" (PRIMEIRA..., 2020).

Se raça e gênero não são consideradas variáveis relevantes na coleta e divulgação de dados sobre a pandemia, por que esses estados deram visibilidade às mulheres negras para dar início à campanha de vacinação? Pode-se inferir que pertencimento racial e de gênero são incorporados pelos estados como estratégia de marketing para agregar valor social ao discurso político (SODRÉ, 2015; LEONG, 2013). Esse debate pode ser aprofundado pela perspectiva do capitalismo racial, que segundo Leong (2013) é "o processo em que indivíduos brancos e instituições predominantemente brancas utilizam pessoas não brancas para adquirir valor social e econômico" (p. 2152, tradução nossa).

Entendo, portanto, esse processo como um jogo, no qual as instituições brancas e masculinas têm o poder se apropriar da raça e gênero com intenção de mostrar-se diversa, menos racistas e machistas, valorizando as mulheres negras. Contudo, a maioria delas não segue as determinações estabelecidas pela Política Nacional de Saúde Integral da População Negra e pelo Estatuto da Igualdade Racial. Isso significa que as identidades racial e de gênero são usadas para aquisição de visibilidade política e social sem, no entanto, levarem a medidas para enfrentamento do racismo e promoção da igualdade neste momento de crise sanitária. Embora os estados e municípios tenham autonomia na gestão da pandemia, o governo federal tem um papel central na gestão das medidas e na orientação dos entes federados - no que falha, sobretudo, no trato da questão racial e de gênero.

\section{REFERÊNCIAS}

AHMED, Mohamed H. Black and Minority Ethnic (BAME) Alliance against Covid-19: one step forward. Journal of Racial and Ethnic Health Disparities, [s. I.], v. 7, n. 5, p. 822-828, out. 2020. DOI: http://dx.doi.org/10.1007/ S40615-020-00837-0. Disponível em: https://link.springer.com/article/10.1007\%2Fs40615-020-00837-0. Acesso em: 4 jun. 2021.

BRASIL. Ministério da Saúde. Portaria n. 992, de 13 de maio de 2009. Institui a Política Nacional de Saúde Integral da População Negra. Diário Oficial da União, Brasília, DF, 13 maio 2009. Disponível em: http:// bvsms.saude.gov.br/bvs/saudelegis/gm/2009/prt0992_13_05_2009.html. Acesso em: 7 mar. 2021.

BRASIL. Ministério da Mulher, da Família e dos Direitos Humanos. Estatuto da Igualdade Racial: Lei no 12.288, de 20 de julho de 2010. E-book. Disponível em: https://www.gov.br/mdh/pt-br/navegue-por-temas/ igualdade-etnico-racial/Estatuto_Igualdade_Racial_.pdf. Acesso em: 7 mar. 2021. 
COVID: primeira idosa vacinada no Rio vive em abrigo do Governo do Estado. RJ.gov, Rio de Janeiro, 18 jan. 2021. Notícias. Disponível em: http://www.ri.gov.br/NoticiaDetalhe.aspx?id noticia=11617\&pl=covid:primeira-idosa-vacinada-no-rio-vive-em-abrigo-do-governo-do-estado. Acesso em: 4 jun. 2021.

CALDWELL, Kia Lilly; Araújo, Edna Maria de. Covid-19 is deadlier for black Brazilians, a legacy of structural racism that dates back to slavery. The Conversation, Waltham, 10 jun. 2020. Disponível em: https://theconversation.com/covid-19-is-deadlier-for-black-brazilians-a-legacy-of-structural-racismthat-dates-back-to-slavery-139430. Acesso em 7 mar. 2021.

ETOWA, Josephine; HYMAN, llene. Unpacking the health and social consequences of COVID-19 through a race, migration and gender lens. Canadian Journal of Public Health, [s. I.], v. 112, p. 8-11, 2021. DOI: https:// doi.org/10.17269/s41997-020-00456-6. Disponível em: https://link.springer.com/article/10.17269/s41997020-00456-6. Acesso em: 4 jun. 2021.

GOMES, Nilma Lino. Questão racial e o novo coronavírus no Brasil. Friedrich Ebert Stifung, São Paulo, 3 jul. 2020. Análise. Disponível em: https://brasil.fes.de/detalhe/a-questao-racial-e-o-novo-coronavirus-no-brasil. Acesso em: 4 jun. 2021.

GOVERNO inicia Campanha Nacional de Vacinação contra a Covid-19 no Espírito Santo. Governo ES, Vitória, 18 jan. 2021. Notícia. Disponível em: https://www.es.gov.br/Noticia/governo-inicia-campanhanacional-de-vacinacao-contra-a-covid-19-no-espirito-santo. Acesso em: 4 jun. 2021.

LEONG, Nancy. Racial capitalism. Harvard Law Review, Cambridge, v. 26, n. 8, p. 2151-2226, jun. 2013. Disponível em: https://harvardlawreview.org/wp-content/uploads/pdfs/vol126 leong.pdf. Acesso em: 4 jun. 2021.

LOPES, Fernanda; WERNECK, Jurema. Saúde da População Negra: da conceituação às políticas públicas de direito. In: WERNECK, Jurema. (Org.). Mulheres Negras: um olhar sobre as lutas sociais e as políticas públicas no Brasil. Rio de Janeiro: Criola, 2009. p. 5-23.

MINAS Gerais inicia a maior operação de vacinação da sua história. Agência Minas, Belo Horizonte, 19 jan. 2021. Rádio. Disponível em: http://agenciaminas.mg.gov.br/multimidia/audio/radio-minas-gerais-inicia-amaior-operacao-de-vacinacao-da-sua-historia. Acesso em: 4 jun. 2021.

ORGANIZAÇÃO DAS NAÇÕES UNIDAS (ONU). ONU alerta para impacto desproporcional da COVID-19 sobre minorias raciais e étnicas. Brasília, DF, 2 jun. 2020. Disponível em: https://brasil.un.org/pt-br/85928-onu-alertapara-impacto-desproporcional-da-covid-19-sobre-minorias-raciais-e-etnicas. Acesso em: 20 fev. 2021.

PRIMEIRA vacinada do país, enfermeira Mônica Calazans ajuda a salvar vidas em SP. Portal do Governo de São Paulo, São Paulo, 17 jan. 2021. Releases. Disponível em: https://www.saopaulo.sp.gov.br/sala-deimprensa/release/primeira-vacinada-do-pais-enfermeira-monica-calazans-ajuda-a-salvar-vidas-em-sp-2/. Acesso em: 4 jun. 2021.

SANTOS, Hebert Luan Pereira Campos dos; MACIEL, Fernanda Beatriz Melo; SANTOS, Kênia Rocha; CONCEIÇÃO, Cídia Dayara Vieira Silva da et al. Necropolítica e reflexões acerca da população negra no contexto da pandemia da Covid-19 no Brasil: uma revisão bibliográfica. Ciência e Saúde Coletiva, Rio de Janeiro, v. 25, supl. 2, p. 4211-4224, 2020. DOI: https://doi.org/10.1590/1413-812320202510.2.25482020. Disponível em: https://www.scielo.br/j/csc/a/5FLQN6ZV5yYPKv6bv4fTbVm/?lang=pt. Acesso em: 4 jun. 2021.

SARAIVA, Adriana. Trabalho, renda e moradia: desigualdades entre brancos e pretos ou pardos persistem no país. Agência IBGE de Notícias, Rio de Janeiro, 12 nov. 2021. Estatísticas Sociais. Disponível em: https:// agenciadenoticias.ibge.gov.br/agencia-noticias/2012-agencia-de-noticias/noticias/29433-trabalho-rendae-moradia-desigualdades-entre-brancos-e-pretos-ou-pardos-persistem-no-pais. Acesso em: 4 jun. 2021.

STARR, Lauren T.; O'CONNOR, Nina R.; MEGHANI, Salimah H. Improved serious illness communication may help mitigate racial disparities in care among black Americans with Covid-19. Journal of General Internal Medicine, Filadélfia, v. 36, n. 4, p. 1071-1076, abr. 2021. DOI: https://dx.doi. org/10.1007\%2Fs11606-020-06557-9. Disponível em: https://www.ncbi.nlm.nih.gov/pmc/articles/ PMC7814859/. Acesso em: 5 jun. 2021.

SODRÉ, Muniz. Claros e escuros: identidade, povo, mídia e cotas no Brasil. Petrópolis: Vozes, 2015. 